\title{
CONTROLE BIOLÓGICO: HELOBDELLA TRISERIALIS LINEATA BLANCHARD, 1849 (HIRUDINEA: GLOSSIPHONIDAE) SOBRE BIOMPHALARIA GLABRATA SAY, 1818 (MOLLUSCA: PLANORBIDAE), EM LABORATÓRIO *
}

Carlos Tito Guimarães **

Cecília Pereira de Souza***

Rotraut A.G.B. Consoli ***

Maria de Lourdes L. de Azevedo *****

\begin{abstract}
GUIMARÃes, C. T. et al. Controle biológico: Helobdella triserialis lineata Blanchard, 1849 (Hirudinea: Glossiphonidae) sobre Biomphalaria glabrata Say, 1818 (Mollusca: Planorbidae), em laboratório. Rev. Saúde públ., S. Paulo, 17:481-92, 1983.
\end{abstract}

RESUMO: A predação de Helobdella triserialis lineata Blanchard, 1849 sobre desovas e exemplares de Biomphalaria glabrata Say, 1818, foi acompanhada visando por um lado verificar a extensão desta predação sobre criações deste molusco em laboratório e, por outro, avaliar a possível utilização desta sanguessuga no controle biológico de planorbíneos hospedeiros intermediários da esquistossomose mansoni em condições naturais. Nas condições do experimento, H. t. lineata mostrou-se eficaz predadora de exemplares de B. glabrata recém-eclodidos, jovens e adultos com diâmetro de até $10 \mathrm{~mm}$. Não ocorreu predação sobre desovas. Observou-se uma aceleração do crescimento e uma redução na oviposição dos planorbíneos mantidos juntos com os hirudineos. Foram feitas algumas observações sobre a biologia de H. t. lineata e sugeridas medidas de controle deste hirudíneo em criações de planorbíneos de laboratório.

UNITERMOS: Helobdella triserialis lineata. Biomphalaria glabrata. Controle biológico. Esquestossomose mansoni.

\section{INTRODŪGAO}

O controle biológico de moluscos de importância médica já de muito vem sendo centado, devendo-se, provavelmente, a pesquisadores japoneses (Myioshima e Juzen Igakkai 10, 1917 e Yuki e Kioto Igakkai 1:, 1919) as primeiras sugestões práticas: larvas de lampirídeos (Coleoptera) e peixes (carpa) no controle dos hospedeiros intermediários do Schistosoma japonicum. Poste- riormente, inúmeras outras espécies - de bactérias a mamiferos - foram tidas como possiveis predadoras e/ou competidoras dos moluscos hospedeiros das esquistossomoses (Michelson 9, 1957 e Ferguson 5, 1972).

A predação de Biomphalaria glabrata por hirudíneos do gênero Helobdella foi anteriormente verificada por Chernin e col.2 (1956), Mc-Annaly e Moore ${ }^{8}$ (1966) e Gonçalves e

Trabalho complementado com auxílio financei ro do "UNDP WORLD BANK"/Special Programme for Research and Training Diseases - WHO.

Do Centro de Pesquisas "René Rachou" - Fundação Oswaldo Cruz - Caixa Postal 1743 - 30000 - Belo Horizonte, MG - Brasil.

*** Do Centro de Pesquisas "René Rachou" e do Departamento de Parasitologia do Instituto de Ciências Biológicas da Universidade Federal de Minas Gerais - 30000 - Belo Horizonte, MG - Brasil.

* Bolsista do Conselho Nacional de Desenvolvimento Científico e Tecnológico (CNPq). 
GUIMARAES, C.T. et al. Controle biológico: Helobaella triserialis lineata Blanchard, 1849 (Hirudinea: Glossiphonidae) sobre Biomphalaria glabrata Say, 1818 (Mollusca: Planorbidae), em laboratório. Rev. Saúde públ., S. Paulo, 17:481-92, 1983.

Pellegrino ${ }^{6}$ (1967). Mais recentemente, Consoli e col. ${ }^{3}$ (1982) iniciaram estudos de laboratório visando verificar a possibilidade da utilização de hirudíneos no controle integrado deste molusco e de larvas de culicideos.

A introdução acidental de sanguessugas do gênero Helobdella no moluscário do Centro de Pesquisas René Rachou, da Fundação Oswaldo Cruz, motivou o presente estudo, pois, além de se adaptarem rapidamente às condições de laboratório, os hirudineos passaram a exercer uma violenta predução sobre os planorbineos, tornando-se em fator limitante da renovação da colônia. Assim, visando avaliar o potencial de predação de Helobdella triserialis lineata sobre desovas e exemplares de $B$. glabrata foram realizados, em laboratório, diferentes experimentos com as duas espécies. Paralelamente, foram coletadas informações sobre biologia e controle dos hirudíneos.

A espécie do hirudíneo focalizada neste trabalho pertence ao ramo Annelida; classe Hirudinea; ordem Rhynchobidellida; familia Glossiphonidae e gênero Helobdella.

\section{MATERIAL E METODOS}

Foram utilizados exemplares e desovas de $B$. glabrata descendentes de exemplares coletados no "Barreiro de Cima" (bairro de Belo Horizonte, MG) e que são mantidos no Laboratório de Testes Biológicos do Centro de Pesquisas "René Rachou" há mais de 10 anos. Nos diferentes experimentos utilizou-se um total de 480 exemplares com diâmetros de 0,$8 ; 1,0 ; 2,0 ; 3,0$;
5,$0 ; 8,0$ e $10,0 \mathrm{~mm}$ e 90 desovas com embriōes de 48 e 144 horas de idade. Os hirudineos tinham, em média, de 6 a $8 \mathrm{~mm}$ de comprimento dor $2 \mathrm{~mm}$ de largura, pesando entre 16 a $20 \mathrm{mg}$. Foram criados em laboratório e descendiam de exemplares acidentalmente introduzidos na criação de moluscos do referido Centro de Pesquisas.

Os experimentos foram desenvolvidos em caixas plásticas retangulares $(31,0 \times 22,0$ $x 9,5 \mathrm{~cm}$ de comprimento, largura e altura, respectivamente) contendo 4 litros de água desclorada, sistema de água corrente e tendo como substrato $50 \mathrm{~cm}^{3}$ de terra laterítica esterilizada acrescida de carbonato de cálcio na proporção de $10 \%$. Estas caixas foram mantidas em sala com temperatura ambiente controlada $\left(25,0 \pm 2,0^{\circ} \mathrm{C}\right)$.

Foram também utilizadas caixas d'água de amianto $(60,0 \times 45,0 \times 40,0 \mathrm{~cm}$ de comprimento, largura e altura, respectivamente) com capacidade para 100 litros, contendo 501 de água de torneira. Como substrato utilizou-se $300 \mathrm{~cm}^{3}$ da terra lateritica anteriormente referida. Estas caixas foram mantidas fora do laboratório expostas a acentuadas variações de temperatura.

O número de hirudíneos adultos nos diferentes experimentos foi mantido constante substituindo-se todo exemplar morto por outro da mesma dimensāo. Como alimento para os planorbíneos utilizou-se folhas frescas de alface.

1. Experimentos com desovas de B. glabrata: Visaram verificar a predação dos hirudineos sobre as desovas. Em 8 caixas plásticas, anteriormente descritas, foi feita a seguinte distribuição:

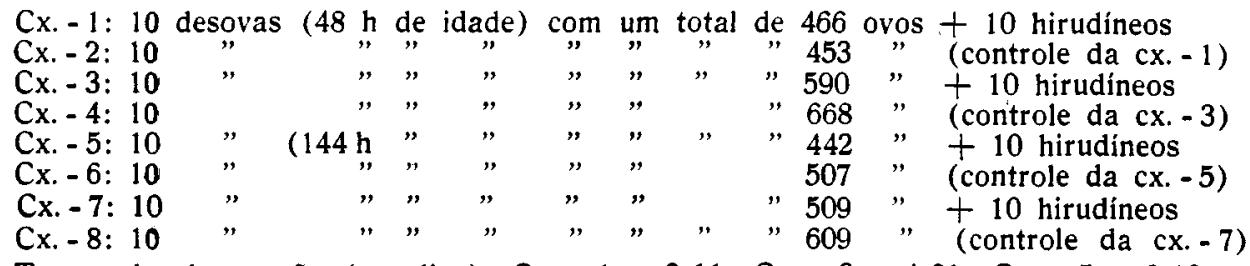

Tempo de observação (em dias): Cxs. -1 e $2: 11$; Cxs. -3 e $4: 21$; Cxs. -5 e 6:10 e Cxs. - 7 e 8:21. 
GUIMARAES, C.T. et al. Controle biologico: Helobdella triserialis lineata Blanchard, 1849 (Hirudinea: Glossiphonidae) sobre Biomphalaria glabrata Say, 1818 (Mollusea: Planorbidae), em laboratório. Rev. Saúde públ., S. Paulo, 17:481-92, 1983.

2. Experimentos com B. glabrata recém-eclodidos, jovens e adultos: Visaram verificar, principalmente, a relação predação/diâmetro dos planorbíneos.
Acompanhou-se também o periodo da predação. Em 14 caixas plásticas foi feita a seguinte distribuição:

$$
\begin{aligned}
& \text { Cx. - 9:20 B. glabrata (diâmetro < que } 1 \mathrm{~mm} \text { ) }+10 \text { hirudineos } \\
& \text { Cx. 10: } 20 " \text { " " (controle da cx. -9) } \\
& \text { Cx. -11: 20" } "(\text { com } 1 \mathrm{~mm} \text { de diâmetro) + } 10 \text { hirudíneos } \\
& \text { Cx. - 12: } 20 " \text { " " " (controle da cx. - 11) } \\
& \text { Cx. 13: } 20 " \quad " 2 \quad \text { " } \quad \text { + } 10 \text { hirudíneos } \\
& \text { Cx. -14: } 20 " \quad " \quad " \quad \text { (controle da cx. - 13) } \\
& \text { Cx. }-15: 20 " \quad " 3 \text { " } " \quad+10 \text { hirudineos } \\
& \text { Cx. }-16: 20 " \quad " \quad " \quad \text { " (controle da cx. 15) } \\
& \text { Cx. - 17: } 20 " \quad " 5 \quad " \quad \text { " } \quad+10 \text { hirudineos } \\
& \text { Cx. }-18: 20 " \quad " \quad " \quad " \quad \text { (controle da } \mathrm{cx},-17 \text { ) } \\
& \text { CX. 19: } 20 " \text { " } " 8 \text { " } 8 \text { hirudineos } \\
& \text { CX. }-20: 20 " \text { " " " } " \text { (controle da cx. -19) } \\
& \text { Cx. -21: } 20 " \quad " 10 \quad \text { " } \quad \text { + } 10 \text { hirudíneos } \\
& \text { Cx. - 22: } 20 " \quad " \quad " \quad \text { (controle da cx. - 21) }
\end{aligned}
$$

Tempo de observação (em dias: Cxs. 9, 10, 11 e 12:1; Cxs. 13, 14, 15 e 16:2; Cxs. 17 e 18:6; Cxs. 19 e 20:83 e Cxs. 21 e 22: 38.

3. Experimentos com B. glabrata adultos: Visaram verificar o crescimento e a oviposição dos planorbíneos em presença de hirudineos. Em 4 caixas plásticas foi feita a seguinte distribuição:

$$
\begin{aligned}
& +10 \text { hirudineos } \\
& \text { (controle da cx. }-23) \\
& +10 \text { hirudineos } \\
& \text { (controle da cx. }-25)
\end{aligned}
$$

Obs.: O número de planorbineos foi mantido constante substituindo-se todo exemplar morto por outro da nesma dimensão. Dia riamente eram feitas contagens de desovas e de 15 em 15 dias os planorbíneos eram medidos. Tempo de observação: 90 dias.

\section{Experimentos em ambientes maiores (caixas d'água de amianto) -}

4.1 Visaram verificar a predação de $H$.

t. lineata sobre desovas e exemplares de
B. glabrata em ambientes maiores e com variações de temperatura. Em 5 caixas d'água, anteriormente descritas, foi feita a seguinte distribuição:

Cx. - I: 10 hirudíneos + 20 B. glabrata (2 a $5 \mathrm{~mm}$ de diâmetro)

CX. - Il: $10 \quad$ + $20 " \quad$ (8 a $12 \mathrm{~mm}$ de diâmetro $)$

CX. - III: $10 \quad+20, \quad$ " (14 a $20 \mathrm{~mm}$ de diâmetro $)$

Cx. - IV: $10 " \quad+10$ desovas de $B$. glabrata (48 horas de idade) com os seguintes números de ovos/desova: $51,52,59,61,68,72,81,88$ e 91 .

Cx. - V: 20 B. glabrata com diâmetros de 2 a $20 \mathrm{~mm}$ (controle).

Tempo de observação: 98 dias. 
GUIMARAES, C.T. et al. Controle blologico: Helobdella triserialis lineata Blanchard, 184! (Hirudinea: Glossiphonidae) sobre Biomphal aria glabrata Say, 1818 (Mollusca: Planorbidae) em laboratório. Rev. Saúde públ., S. Paulo, 17:481-92, 1983.

4.2 Visaram acompanhar o crescimento e a oviposição dos planorbíneos em presença dos hirudineos nos ambientes maiores. Em 4 caixas d'água foi feita a seguinte distri buição:

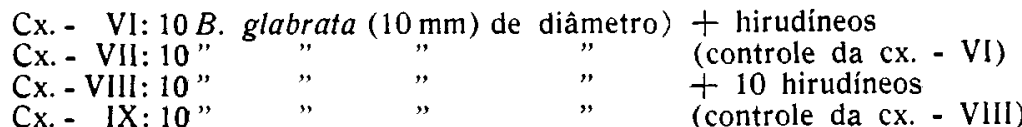

CX. - IX: $10 " \quad " \quad$ " " $"$ (controle da cx. - VIII)

Obs.: As desovas de $B$. glabrata ovipostas nas caixas VI e VII eram contadas e retiradas diariamente; as caixas VIII e IX eram contadas, marcadas (para evitar recontagens) e mantidas nas caixas. Tempo de observação: 152 dias.

Nos experimentos em que se compararam o crescimento e a oviposição dos planorbineos em presença e ausência de $H$. $t$. lineata, os pares de médias foram comparados pelo teste " $t$ " de Student.

5. Observaçöes sobre alguns aspectos da biologia de $H$. $t$. lineata

Em 12 caixas plásticas com um exemplar de $H$. $t$. lineata em cada foram feitas algumas observações sobre longevidade, duração do ciclo ovo a ovo, número de ovos por desova, intervalos entre oviposições, período de incubação dos ovos, hábitos alimentares entre outras.

\section{RESULTADOS}

1. Experimentos com desovas de B. glabrata

Nas condiçöes do experimento, os hirudíneos não predaram as desovas de $B$. glabrata, atacando, entretanto, os planorbíneos recém-eclodidos logo que saiam dos ovos. Observou-se que nas desovas com 48 t de idade expostas aos hirudíneos, o desenvolvimento embrionário foi normal até a eclosão, embora em diversas oportunidades fossem observadas sanguessugas sobre as mesmas. Observação análoga foi feita em relação às desovas com $144 \mathrm{~h}$ de idade.

Assim, a Tabela 1 mostra a predação dos hirudineos sobre os exemplares recém-eclo-

T A B E L A 1

Atividade predatória de Helobdella triserialis lineata sobre exemplares recém-eclodidos de Biomphalaria glabrata, em ambientes menores (caixas plásticas), em laboratório (Belo Horizonte, MG. - Março/1980).

\begin{tabular}{|c|c|c|c|c|c|c|c|c|}
\hline \multirow{3}{*}{$\begin{array}{l}\text { Ne da } \\
\text { caixa }\end{array}$} & \multirow{3}{*}{$\begin{array}{c}\text { No de } \\
\text { hirudíneos }\end{array}$} & \multicolumn{5}{|c|}{ Biomphalaria glabrata } & \multirow{3}{*}{$\begin{array}{l}\text { Período } \\
\text { de } \\
\text { predação }\end{array}$} & \multirow{3}{*}{$\begin{array}{c}\text { Periodo } \\
\text { de } \\
\text { observaçă } \\
\text { (em dias) }\end{array}$} \\
\hline & & \multicolumn{3}{|c|}{ Desovas } & \multicolumn{2}{|c|}{$\begin{array}{c}\text { Exemplares } \\
\text { sobreviventes }\end{array}$} & & \\
\hline & & Número & $\begin{array}{c}\text { Idade } \\
\text { (em horas) }\end{array}$ & $\begin{array}{l}\text { No de } \\
\text { ovos }\end{array}$ & $\mathrm{N}^{\circ}$ & $\%$ & & \\
\hline 1 & 10 & 10 & 48 & 466 & 4 & 0,9 & $79^{\circ} 11^{\circ} \mathrm{dia}$ & 15 \\
\hline 2 & - & 10 & 48 & 453 & 453 & 100,0 & - & 15 \\
\hline 3 & 10 & 10 & 48 & 590 & 0 & & $7^{\circ}$ ao $21^{\circ} \mathrm{dia}$ & 25 \\
\hline 4 & - & 10 & 48 & 668 & 665 & 99,6 & - & 25 \\
\hline $\mathbf{5}$ & 10 & 10 & 144 & 442 & 0 & - & $3^{\circ}$ ao $7 \circ$ dia & 15 \\
\hline 6 & - & 10 & 144 & 507 & 507 & 100,0 & - & 15 \\
\hline 7 & 10 & 10 & 144 & 509 & 0 & - & $20^{\circ}$ a $15 \circ$ dia & 18 \\
\hline 8 & - & 10 & 144 & 609 & 609 & 100,0 & - & 18 \\
\hline
\end{tabular}


GUIMARAES, C.T. et al. Controle biológico: Helobdella triserialis lineata Blanchard, 1849 (Hirudinea: Glassiphonidae) sobre Biomphalaria glabrata Say, 1818 (Mollusca: Planorbidae), em laboratório. Rev. Saúde públ., S. Paulo, 17:481-92, 1983.

didos de $B$. glabrata. Ressaite-se que a mortalidade no grupo experimental chegou a $99,6 \%$, enquanto no grupo controle nāo atingiu $0,5 \%$.

2. Experimentos com exemplares recém-eclodidos, jovens e adultos de B. glabrata:

a) Em ambientes menores (caixas plásticas). De acordo com os dados da Tabela 2, os exemplares de $B$. glabrata com diâmetros menor e igual a $1 \mathrm{~mm}$ foram totalmente predados pelos hirudineos em $24 \mathrm{~h}$; os com 2 e $3 \mathrm{~mm}$ entre o 10 e o $2^{\circ}$ dias; os com $5 \mathrm{~mm}$ entre o $2 \%$ e o $6 \%$ dia e os com $8 \mathrm{~mm}$ entre o $2^{\circ}$ c o 7 dia. A predação sobre os exemplares com $10 \mathrm{~mm}$ ocorreu entre o $2^{\circ}$ e o $12^{\circ}$ dia. Neste caso, entretanto, como também ocorrido com os exemplares de $8 \mathrm{~mm}$, a predação foi bastante atenuada, pois ao final de 90 dias de observaçōes, dos 20 planorbineos iniciais, $15(75,0 \%)$ permaneciam vivos.

T A B E L A

Atividade predatória de Helobdella triserialis lineata sobre Biamphalaria glabrata de diferentes diámetros em ambientes menores (caixas plásticas), em laboratório (Belo Horizonte, MG., março - Malo/1981).

\begin{tabular}{|c|c|c|c|c|c|c|c|}
\hline \multirow{3}{*}{$\begin{array}{l}\text { No da } \\
\text { caixa }\end{array}$} & \multirow{3}{*}{$\begin{array}{l}\text { No de } \\
\text { hirudíneos }\end{array}$} & \multicolumn{4}{|c|}{ Biomphalaria glabrata } & \multirow{3}{*}{$\begin{array}{l}\text { Período } \\
\text { de } \\
\text { predagão }\end{array}$} & \multirow{3}{*}{$\begin{array}{l}\text { Periodo } \\
\text { de } \\
\text { observação } \\
\text { (em dias) }\end{array}$} \\
\hline & & \multirow{2}{*}{$\begin{array}{l}\text { No de } \\
\text { exem- } \\
\text { plares }\end{array}$} & \multirow[t]{2}{*}{$\begin{array}{l}\text { Diâmetro } \\
\text { (em mm) }\end{array}$} & \multicolumn{2}{|c|}{$\begin{array}{l}\text { Exemplares } \\
\text { sobreviventes }\end{array}$} & & \\
\hline & & & & Número & $\%$ & & \\
\hline
\end{tabular}

\begin{tabular}{|c|c|c|c|c|c|c|c|}
\hline 9 & 10 & 20 & $<1$ & 0 & - & 24 horas & 1 \\
\hline 10 & - & 20 & $<1$ & 20 & 100,0 & - & 1 \\
\hline 11 & 10 & 20 & 1 & 0 & - & 24 horas & 1 \\
\hline 12 & - & 20 & 1 & 20 & 100,0 & - & 1 \\
\hline 13 & 10 & 20 & 2 & 0 & - & 10 ao $2^{\circ} \mathrm{dla}$ & 2 \\
\hline 14 & - & 20 & $\overline{2}$ & 20 & 100,0 & - & 2 \\
\hline 15 & 10 & 20 & $\mathbf{3}$ & 0 & - & $1^{\circ}$ ao $2^{\circ} \mathrm{dia}$ & 2 \\
\hline 16 & - & 20 & 3 & 20 & 100.0 & - & 2 \\
\hline 17 & 10 & 20 & 5 & 0 & - & 20 ao 60 dia & 6 \\
\hline 18 & - & 20 & 5 & 20 & 100,0 & - & 6 \\
\hline 19 & 10 & 20 & 8 & 10 & 50,0 & $2 \circ$ ao $7 \circ$ dia & 83 \\
\hline 20 & - & 20 & 8 & 16 & 80,00 & - & 83 \\
\hline 21 & 10 & 20 & 10 & 15 & 75,0 & $2^{\circ}$ ao $12^{\circ}$ dia & 38 \\
\hline 22 & - & 20 & 10 & 20 & 100,0 & - & 38 \\
\hline
\end{tabular}

Observou-se uma redução na velocidade de predação diretamente proporcional ao aumento de diâmetro dos planorbineos.

b) Em ambientes maiores (caixas d'água de amianto). Estes experimentos visaram verificar principalmente se as interações ocorridas nas caixas plásticas (ambientes menores) ocorreriam também nos ambientes maiores onde os contatos entre pla- norbineos e hirudineos seriam, presumivelmente, menos constantes. Por outro lado, procurou-se observar o comportamento das duas espécies quando submetidas a acentuadas variações de temperatura, como ocorre em condições naturais, uma vez que estes experimentos foram desenvolvidos fora do laboratório. Nestas condições, a temperatura do ar 
GUIMARAES, C.T. et al. Controle biológico: Helobdella triserialis lineata Blanchard, 184! (Hirudinea: Glossiphonidae) sobre Biomphalaria glabrata Say, 1818 (Mollusca: Planorbidae), em laboratório. Rev. Saúde públ., S. Paulo, 17:481-92, 1983.

variou de 13,0 a $34,0^{\circ} \mathrm{C}$ e a da água de 15,5 a $24,5^{\circ} \mathrm{C}$.

Assim, os dados contidos na Tabela 3 - confirmando as observaçōes anteriores (caixas plásticas) - mostram também a predação dos hirudíneos sobre os exempla- res menores (diâmetros entre 3 e $9 \mathrm{~mm}$ ) de B. glabrata. Os exemplares maiores (15. 16,20 e $21 \mathrm{~mm}$ de diâmetro) encontrados mortos nas caixas I e III provavelmente também foram predados pelos hirudíneos. Entretanto, neste caso, a hipótese de outras causas-mortis não deve ser desprezada.

\section{T A B E L A 3}

Atividade predatória de Helobdella triserialis lineata sobre Biomphalaria glabrata de diferentes diametros em ambientes maiores (caixas d’água de amianto), em laboratório (Belo Horizonte, MG., Setembro-Dezembro/1981).

\begin{tabular}{|c|c|c|c|c|c|c|c|c|}
\hline \multirow{3}{*}{ Semanas } & \multicolumn{8}{|c|}{ Biomphalaria glabrata mortas } \\
\hline & \multicolumn{2}{|c|}{$\begin{array}{c}\text { Caixa - I* } \\
\text { (com hirudineos) }\end{array}$} & \multicolumn{2}{|c|}{$\begin{array}{c}\text { Caixa - II* } \\
\text { (com hirudineos) }\end{array}$} & \multicolumn{2}{|c|}{$\begin{array}{c}\text { Caixa - III* } \\
\text { (com hirudineos) }\end{array}$} & \multicolumn{2}{|c|}{$\begin{array}{c}\text { Caixa }-V^{*} \\
\text { (sem hirudineos) }\end{array}$} \\
\hline & Ne & $\begin{array}{l}\text { Diametro } \\
\text { (em mm) }\end{array}$ & No & $\begin{array}{l}\text { Diametro } \\
\text { (em mm) }\end{array}$ & No & $\begin{array}{l}\text { Diametro } \\
\text { (em mm) }\end{array}$ & No & $\begin{array}{l}\text { Diâmetro } \\
\text { (en } \mathrm{mm} \text { ) }\end{array}$ \\
\hline $1 \mathfrak{a}$ & 6 & $3 ; 3 ; 4 ; 4 ; 4$ e 5 & 1 & 8 & 0 & - & 0 & - \\
\hline $2 a$ & 3 & $5 ; 5$ e 6 & 1 & $\mathbf{9}$ & 0 & 一 & 0 & - \\
\hline $\mathbf{3 a}$ & 3 & $6 ; 7$ e 7 & $\mathbf{0}$ & - & 0 & 一 & 0 & - \\
\hline $4 a$ & 1 & 9 & 0 & - & 0 & - & 0 & - \\
\hline $5 \mathfrak{a}$ & 0 & - & 0 & 一 & 0 & - & 0 & - \\
\hline $6 a$ & 3 & $7 ; 8$ e 8 & 0 & - & 0 & - & 0 & - \\
\hline $7 \mathfrak{9}$ & 2 & 7 e 8 & 0 & -- & 0 & - & 0 & - \\
\hline 8 & 0 & - & 0 & - & 0 & - & 0 & - \\
\hline $9 \mathrm{a}$ & 1 & 15 & 0 & - & 0 & - & 0 & - \\
\hline $10 a$ & 0 & - & 0 & - & 1 & 21 & 0 & - \\
\hline 11a & 0 & - & 0 & - & 1 & 16 & 0 & - \\
\hline $12 \mathrm{a}$ & 0 & - & 0 & - & 0 & - & 0 & - \\
\hline $13 a$ & 0 & $\longrightarrow$ & 0 & - & 1 & 20 & 0 & - \\
\hline 14 & 0 & - & 0 & - & 0 & - & 0 & - \\
\hline Total & 19 & & 2 & & 3 & & 0 & \\
\hline
\end{tabular}

* No de exemplares iniciais nas caixas: B. glabrata - $20 /$ H.t.lineata -

10 Diámetros de $B$. glabrata no inicio do experimento: $\mathrm{Cx}$. I -2 a $5 \mathrm{~mm} / \mathrm{Cx}$. II 8 a $12 \mathrm{~mm} / \mathrm{Cx}$. III - 14 a $20 \mathrm{~mm}$ e Cx. V -2 a $20 \mathrm{~mm}$ (controle).

E importante relatar que ao final de 14 semanas (duração do experimento) encontrou-se um exemplar de $B$. glabrata $(17 \mathrm{~mm}$ de diâmetro) na caixa $-1 ; 18(15 \mathrm{a} 22 \mathrm{~mm})$ na caixa - II; 64 (2 a $23 \mathrm{~mm})$ na caixa -IIl e 425 exemplares ( 2 a $22 \mathrm{~mm}$ ) na caixa - V. Na caixa IV, onde no inicio do experimento foram colocadas 10 desovas de $B$. glabrata e 10 exemplares de $H$. $t$. lineata, não foi encontrado nenhum planorbíneo.
3. Experimenios com B. glabrata adultos (crescimento e oviposição em presença de hirudineos). Os dados contidos na Tabela 4 mostram que os exemplares de $B$. glabrata que conviviam com os hirudineos cresceram mais que o grupo controle (sem hirudineos) tanto nas caixas plásticas (ambientes menores), mantidas dentro do laboratório com temperatura controlada $\left(25,0 \pm 2,0^{\circ} \mathrm{C}\right)$, quanto nas caixas d'água (ambientes 
GUIMARAES, C.T. et al. Controle biológico: Helobdella triserialis lineata Blanchard, 1849 (Hirudinea: Glossiphonidae) sobre Biomphalaria glabrata Say, 1818 (Mollusca: Planorbidae), em laboratório. Rev. Saúde públ., \&. Paulo, 17:481-92, 1983.

maiores) mantidas fora do laboratório com variações na temperatura. Observa-se que a diferença entre as médias dos diâmetros dos planorbíneos em presença e ausência de hirudineos é altamente significativa $(\alpha=0,01)$, exceto para os intervalos entre 61 e 75 dias nas caixas plásticas e um a 15 dias nas caixas d'água, nos quais a significância foi ao nivel de $5 \%$.
Por outro lado, a Tabela 5 mostra uma redução na oviposição dos planorbineos que conviviam com os hirudineos tanto nas caixas plásticas, quanto nas caixas d'água. Observa-se que o número médio de desovas de $B$. glabrata é significativamente menor em presença de $H$. $t$. lineata $(\alpha=0,01)$ tanto nos ambientes maiores quanto nos menores, exceto nos intervalos entre $46 \mathrm{e}$

T A B E L A 4

Diâmetros médios (em $\mathrm{mm}$ ) e desvios padrões ( $\chi \pm \mathrm{s}$ ) de exemplares de Biomphalaria glabrata mantidos em presença e ausência de Helobdella triserialis lineata em caixas plásticas (ambientes menores) e caixas d'água (ambientes malores), em laboratórío. (Belo Forizonte, MG., Dezembro/1981 - Agosto/1982).

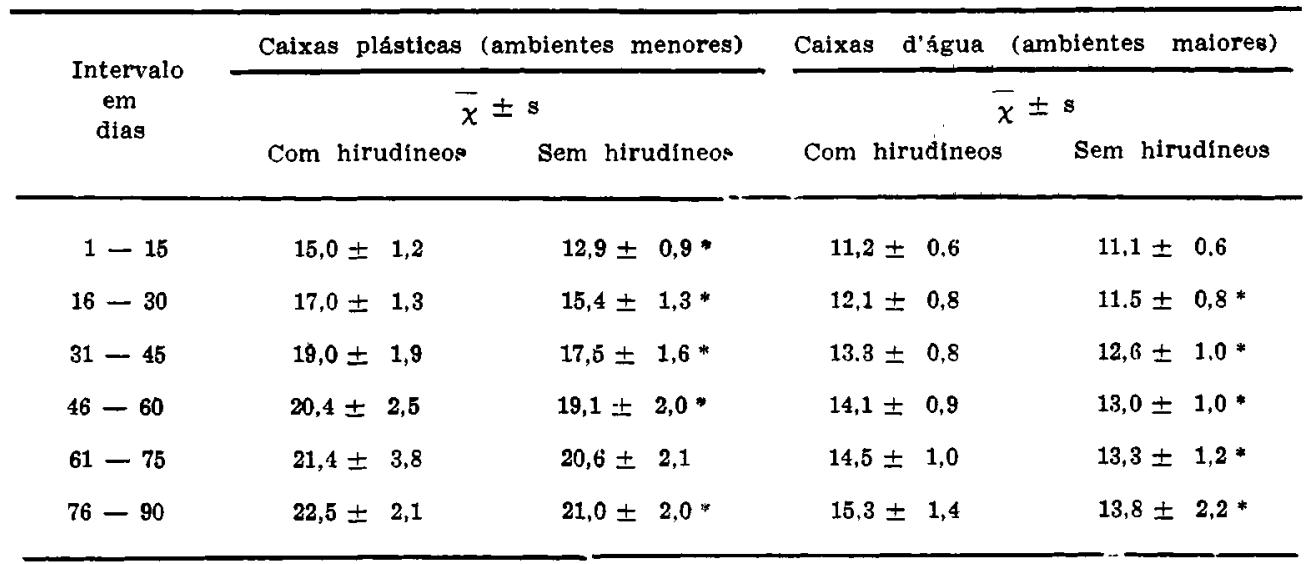

* Diferenças entre as médias significativas ao nivel de $\alpha=0.01$.

60 dias nas caixas plásticas e 61 a 75 e 76 a 90 dias nas caixas d'água quando a significância das diferenças atingiu o nivel de $\alpha=0,05$.

Outra observação, feita apenas nas caixas plásticas, mostrou que o número médio de ovos depositados pelos planorbineos em presença dos hirudineos foi significativamente menor $(\alpha=0,01)$ que no controle, exceto nos intervalos entre 31 e 45 dias $(\alpha=0,05)$ e 46 e 60 dias quando não foi estatisticamente significativo.

\section{Algumas observafōes de laboratório cobre biologia de $\mathrm{H}$. t. lineata.}

Em relação à reprodução, observou-se que os ovos ficam aderidos à superfície ventral do genitor, sendo de cor cinza logo que ovipostos e escurecendo posteriormente. $O$ período de incubação dos ovos foi de 5 a 6 dias à temperatura controlada $\left(25,0 \pm 2,0^{\circ} \mathrm{C}\right)$. Os hirudineos recém-eclodidos também permanecem aderidos à superfície ventral do genitor por cerca de 6 dias. Em 6 desovas observadas foram contados $19,22,24,27,32$ e 47 ovos, respectiva- 
GUIMARAES, C.T. et al. Controle biológico: Helobdella triserialis lineata Blanchard, 1849 (Hirudinea: Glossiphonidae) sobre Biomphal aria glabrata Say, 1818 (Mollusca: Planorbidae), em laboratório. Rev. Saúde públ., S. Paulo, 17:481-92, 1983.

T A B E L A 5

Número médios de desovas de Bimphalaria glabrata por dia e desvios padrões $(\bar{\chi} \pm s)$ $\mathrm{cm}$ presença e ausência de Helobdella triserialis lineata em caixas plásticas (ambientes menores) e caixas d'água (ambientes maiores), em laboratório (Belo Horizonte, MG.. Dezembro/1981 - Agosto/1982).

\begin{tabular}{|c|c|c|c|c|}
\hline \multirow{3}{*}{$\begin{array}{c}\text { Intervalo } \\
\text { em } \\
\text { dias }\end{array}$} & \multicolumn{2}{|c|}{ Caixas plásticas (ambientes menores) } & \multicolumn{2}{|c|}{ Caixas d'água (cmbientes maiores) } \\
\hline & \multicolumn{2}{|c|}{$\bar{x} \pm s$} & \multicolumn{2}{|c|}{$\bar{x} \pm s$} \\
\hline & Com hirudineos & Sem hirudineos & Com hirudíneos & Sem hirudineos \\
\hline $1-15$ & - & $4,4 \pm 4,0^{*}$ & $2,0 \pm 1,8$ & $8,3 \pm 5,4 *$ \\
\hline $16-30$ & $0,5 \pm 1,0$ & $16,5 \pm 13,0^{*}$ & $4,1 \pm 1,5$ & $11,0 \pm 3,0^{*}$ \\
\hline $31-45$ & $5,1 \pm 4,8$ & $24,4 \pm 17,1 *$ & $5,0 \pm 2,1$ & $8,6 \pm 2,4^{*}$ \\
\hline $46-60$ & $11,9 \pm 11,3$ & $24,1 \pm 21,3$ & $6.7 \pm 1.9$ & $7,3 \pm 2,6$ \\
\hline $61-75$ & $8,8 \pm 6.6$ & $19,1 \pm 12,1 *$ & $3,8 \pm 3,2$ & $8,3 \pm 5,4$ \\
\hline $76-90$ & $6,3 \pm 4,0$ & $19,5 \pm 10,5 *$ & $6,2 \pm 4,4$ & $10,1 \pm 4,5$ \\
\hline
\end{tabular}

"Diferenças cntre as médias significativas an nível de $\alpha=0,01$.

mente. $O$ intervalo entre uma oviposição e outra variou de 30 a 60 dias e o comprimento dos exemplares que desovaram foi de 8 a $13 \mathrm{~mm}$.

Quanto aos hábitos alimentares, os hirudíneos se alimentaram de fígado de boi $e$ de galinha apresentando, no entanto, alta mortalidade. Alimentaram-se naturalmente de exemplares recém-eclodidos e jovens de B. glabrata e de larvas de culicídeos. Observou-se que a alimentação pode ser feita tanto durante o dia quanto a noite e que a resistência ao jejum pode chegar a 2 meses.

Em relação à longevidade, o hirudineo que mais sobreviveu em laboratório morreu com 10 meses e 23 dias de idade. Durante este periodo ele desovou 4 vezes e atingiu o comprimento de $13 \mathrm{~mm}$, o maior registrado nas condições do experimento.

\section{DISCUSSÃO}

Helobdella triserialis lineata é um anelídeo de água doce comumente encontrado em áreas vizinhas à Belo Horizonte, $M G$, em diferentes criadouros (córregos, lagoas, valas entre outros). Sua introdução em moluscários é, via de regrá, acidental, com os primeiros exemplares vindos aderidos às conchas dos moluscos capturados no campo, plantas aquáticas, folhas de alface, etc. Sua adaptação às condições de laboratório é rápida, aparecendo logo os sinais de sua presença: redução acentuada na produção de caramujos em decorrência da alta mortalidade de recém-eclodidos e jovens.

No presente estudo detectou-se uma violenta predação de $H$. $t$. lineata sobre exemplares recém-eclodidos e jovens (diâmetro até $9 \mathrm{~mm}$ ) de $B$. glabrata. A predação de exemplares com diâmetro a partir de $10 \mathrm{~mm}$ também foi observada, ocorrendo, no entanto, com menor intensidade. Não houve predação sobre desovas.

De acordo com Gonçalves e Pellegrino ${ }^{6}$ (1967), Helobdella triserialis (provavelmente Helobdella triserialis lineata) preda desde exemplares recém-eclodidos de $B$. glabrata até os com diâmetro de $16 \mathrm{~mm}$. 
GUIMARAES, C.T. et al. Controle biológico: Helobdella triserialis lineata Blanchard, 1849 (Hirudinea: Glossiphonidae) sobre Biomphal aria glabrata Say, 1818 (Mollusca: Planorbidae), em laboratório. Rev. Saúde públ., S. Paulo, 17:481-92, 1983.

Segundo esses autores, 5 hirudíneos da espécie em questão, com $5 \mathrm{~mm}$ de comprimento, predaram em 45 dias: 44 exemplares de B. glabrata de 1 a $2 \mathrm{~mm}$ de diâmetro; 19 de 3 a $4 \mathrm{~mm} ; 6$ de 5 a $10 \mathrm{~mm}$ e 6 de 13 a $16 \mathrm{~mm}$. Chernin e col.2 (1956) afirmam que um outro hirudíneo do mesmo gênero (Helobdella fusca), em condiçōes de laboratório, é um eficaz predador de $B$. glabrata, principalmente jovens. Assinalam ainda esses autores que esta espécie também não preda desovas deste planorbíneo. A conclusões semelhantes chegaram McAnnaly e Moores (1966) trabalhando com Helobdella punctato-lineata também em laboratório.

No presente trabalho, além da predaçăo dos hirudineos sobre $B$. glabrata, duas outras observações chamaram atenção: 0 aumento na velocidade do crescimento e a redução da oviposição daquele planorbíneo quando em presença de $H$. $t$. lineata.

O maior desenvolvimento dos exemplares de $B$. glabrata em presença dos hirudíneos foi observado inicialmente nas caixas plásticas (ambientes menores) e, posteriormente, confirmado nos ambientes maiores (caixas d'água).

Ao que as observações sugerem, este fato poderia ser considerado como uma auto-defesa dos planorbíneos, considerando-se que 'a predação dos hirudíneos, nas condiçōes dos experimentos, foi bem mais acentuada sobre os exemplares menores (diâmetros abaixo de $9 \mathrm{~mm}$ ). O mecanismo deste fenômeno não foi detectado. Todavia, algumas conjecturas podem ser feitas, como, por exemplo, supor-se a eliminaçáo pelos hirudíneos de alguma substância que acelerasse o crescimento dos planorbíneos. Esta hipótese - caso verdadeira - seria respaldada pelo fato de que nas caixas plásticas (4 litros de água) o crescimento dos planorbineos foi bem mais acentuado que nas caixas d'água de amianto (50 litros de água).
Ainda dentro do enfoque da auto-defesa, em diferentes ocasiōes observou-se a saida da água de planorbineos nas caixas em que eles conviviam com os hirudineos. Guimarães 7 (1983) assinalou este mesmo comportamento em populações de $B$. tenagophila quando coabitavam o mesmo aquário com - pilideo Pomacea haustrum.

A redução da oviposição de $B$. glabrata em presença de $H$. t. lineata foi também observada inicialmente nas caixas plásticas (ambientes menores) e, posteriormente, confirmada nos ambientes maiores (caixas d'água de amianto). Também aqui, o mecanismo deste fenômeno não pôde ser identificado, sugerindo-se, como no caso da aceleração do crescimento, a participação de alguma substância secretada pelos hirudíneos. Em relação a este aspecte, El-Hassan 4 (1974) sugeriu a utilização de um planotbídeo (Helisoma tenue) para controlar outros moluscos (Bulinus truncatus e Biomphalaria alexandrina), pois além de muito reprodutivo e adaptável, o $H$. tenue secretaria substâncias que inibiriam a oviposição das referidas espécies.

Os dados do presente trabalho mostram que nas condiçōes do experimento $H$. $t$. lineata é um efetivo predador de $B$. glabrata atuando, principalmente, sobre os exemplares recém-eclodidos e jovens (diâmetro até $9 \mathrm{~mm}$ ), confirmando observações de outros autores: Chernin e col. ${ }^{2}$ (1956), McAnnaly e Moores (1966) e Gonçalves e Pellegrino ${ }^{6}$ (1967).

$O$ encontro de planorbíneos e hirudineos na Natureza, coabitando os mesmos criadouros, sugere a ocorrência de um equilibrio entre as populações das duas espécies. As observaçōes sugerem que o hirudineo em questão, embora predador de planorbineos, não seria um "predador monófago", isto é, que só subsistiria às expensas de uma única presa, no caso os planorbíneos. Para Storer e Usinger (1957), "as sanguessugas são necrófagas, predadoras ou parasitas; algumas alimentam-se de animais mortos e outras aprisionam pequenos vermes, insetos, 
GUIMARAES, C.T. et al. Controle biológico: Helobdella triserialis lineata Blanchard, 184: (Hiruāinea: Glossiphonidae) sobre Biomphalaria glabrata Say, 1818 (Mollusca: Planorbidae), em laboratório. Rev. Saude públ., S. Paulo, 17:481-92, 1983.

larvas, moluscos, etc." É importante ressaltar que, em condições de laboratório, exemplares de $H$. $t$. lineata predaram tanto planorbíneos quanto larvas de culicídeos encontradas na água (Consoli e col. ${ }^{3}, 1982$ ).

Assim, a utilização deste hirudíneo no controle biológico de $B$. glabrata e outros hospedeiros intermediários das esquistossomoses, em condições naturais, deve ser cuidadosamente avaliada. Dentre outros autores, Brumpt ${ }^{1}$ (1941), apesar de ter acompanhado, em laboratório, a destruição de uma cơlônia de caramujos por Helobdella stagnalis, considerou secundário o papel das sanguessugas na destruição de caramujos na Natureza. Para Gonçalves e Pellegrino ${ }^{6}$ (1967), sanguessugas do gênero Helobdella são potencialmente perigosas para as colônias de planorbineos mantidas em laboratório. Chernin e col.2 (1956), afirmam: Whether Helobdella fusca, $H$. punctato-linea$t a$, or indeed any other leech represents a pratical means for the control of Australorbis glabratus under natural conditions remains a moot question which can be resolved only by field trial". E continuam: "There is no doubt, however, that Helobdella fusca is a most effective agent for the control of $A$. glabratus populations in the laboratory". Da mesma forma, McAnnaly e Moore 8 (1966) consideram as sanguessugas um risco em potencial para as colônias de caramujos de laboratório.

Os resultados do presente estudo - confirmando as observações dos autores citados - indicam que a $H$. $t$. lineata, apesar de predar planorbíneos, é mais um problema para as criaçōes destes moluscos em laboratório do que uma solução para seu controle em condiçōes naturais. Todavia, reprisando Chernin e col.2 (1956), a inexistência de experimentos de campo não permitem o fechamento da questão.

Pesquisadores do Laboratório de Ecologia e Controle Biológico do Centro de Pesquisas "René Rachou" da Fundação Oswaldo Cruz estão tentando localizar criadouros de pla- norbineos comprovadamente livres de hiru. díneos. Caso isto seja conseguido, após, c levantamento da dinâmica populacional do: planorbíneos, serão introduzidos ali exem. plares de $H$. t. lineata. Com este procedi. mento talvez seja possivel avaliar, con maior segurança, o verdadeiro papel do: hirudíneos no controle biológico de planor bíneos hospedeiros intermediários da esquis tossomose mansoni em condições naturais.

\section{MEDIDAS DE CONTROLE DE HIRUDINEÓ EM CRIAÇOES DE MOLUSCOS}

A contaminação de criações de moluscu: em laboratório por hirudíneos é sempre un problemá de difícil solução. McAnnaly । Moore 8 (1966) afirmam que medidas come destruição das plantas aquáticas, esterili zação do substrato e aquários e examı microscópico dos caramujos antes de reco. locá-los nos aquários limpos não eliminan a infestação. As medidas a seguir rela. cionadas - testadas com sucesso no Labo. ratório de Testes Biológicos do Centro dı Pesquisas René Rachou da Fundaçã Oswaldo Cruz - poderão auxiliar ne resolução do problema.

19) Exame minucioso do material (moluscos plantas aquáticas, entre outros) coletadc no campo antes de introduzi-lo nos aquários.

20) Exame minucioso semanal dos aquários para deteç̧ão precoce de sanguessugas

3 ?) Lavagem dos aquários contaminados com álcool comercial $\left(96^{\circ} \mathrm{GL}\right)$ que eli. mina as sanguessugas recém-eclodidas jovens e adultas.

40) Eliminação (se possível) de todo ma. terial (moluscos, substrato, plantas aquáticas, entre outros) do(s) aquários(s) contaminado(s).

$\left.5^{\circ}\right)$ Iniciar (sempre que possível) nova criação de moluscos a partir de desovas comprovadamente não contaminadas (examinadas em microscópio estereos. cópico). 
GUIMARAES, C.T. et al. Controle biologico: HIelobdella triserialis lineata Blanchard, 1849 (Hirudinea: Glossiphonidae) sobre Biomphalaria glabrata Say, 1818 (Mollusca: Planorbidae), em laboratório. Rev. Saúde públ., S. Paulo, 17:481-92, 1983.

\section{AGRADECIMENTOS}

Ao Dr. G.L. Hoffman da "Fish Farming Esper. Sta. Usews, Stuttgart", EUA, por sua inestimável ajuda, e ao Dr. R.A. Ringuelet do Instituto de Limnologia da Universidade de La Plata, Argentina, pela identificação dos hirudíneos.

GUIMARAES, C. T. et al. [Blological control: Hełobcclla triserialiśs lineấa Blanchard, 1849 (Hirudinea: Glossiphonidae) over Biomphalaria glabrata Say, 1818 Mollusca: Planorbidae), in laboratory]. Rev. Saúde públ., S. Paulo, 17:481-92, 1983.

ABSTRACT: The predation of Helobdella triserialis lineata Blanchard, 1849 , on eggmasses and specimens of Biomphalaria glabrata Say, 1818 was observed in the laboratory. H. t. lineata was considered an efficient predator on newlyhatched snails, as well as on young and adult specimens with diameter up to 10 millimeters. No predation on eggmasses was observed. Accelerated growth and reduced oviposition were observed in planorbids kept together with the leeches. Some observations were made on the biology of H. t. lineata in experimental conditions, and some measures to control this leech in laboratory colonies of planorbids are suggested.

UNITERMS: Helobdella triserialis lineata. Biomphalaria glabrata. Biological control. Schistosomiasis.

\section{REFERENCIAS BIBLIOGRAFICAS}

1. BRUPT, E. Observations biologiques diverses concernant Planorbis (Australorbis) glabratus hôte intermédiaire de Schistosoma mansoni. Ann. Parasit. hum. comp., $18: 9-45,1941$.

2. CHERNIN, E.; MICHELSON, E.H. \& AUGUSTINE, D.L. Studies on the biological control of schistosome-bearing snails. II - The control of Australorbis glabratus poprlations by the leech, Helobdella fusca, under laboratory conditions. Amer. J. trop. Med. Hyg., 5:308-14, 1956.

3. CONSOLI, R.A.G.B.; SOUZA, C.P. \& GUIMARÃes, C.T. Predação de $\mathrm{He}$ lobdella sp. (Hirudinea; Glossiphonidae) sobre formas imaturas de culicideos e moluscos da espécie Biomphalaria glabrata. In: Congresso da Sociedade Brasileira de Parasitologia, 70, Porto Alegre, 1982. Resumos. Porto Alegre, 1982. p. $8 \%$.

4. EL-HASSAN, A.A.A. Helisoma temue and Physa acuta snails as biological means of control against Bulinus truncatus and Biomphalaria alexandrina, snail in Egypt. In: International Congress of Parasitology, Munchen, 1974. Proceedings. Munchen, 1974. v. 3, p. 1597.
5. FERGUSON, F.F. Biological control of schistosomiasis snails. In: Symposilim of the Future of Schistosomiasis Control, New Orleans, 1972. Proceedings. New Orleans, Tulane University, 1972. p. 85-91.

6. GONÇALVES, M. da G.R. \& PELLEGRINO, J. Predatory activity of Helobdela triserialis (Blanchard, 1849) upon Biomphalaria glabrata under laboratory conditions. J. Parasit., 53:30, 1967.

7. GUIMARAES, C.T. Controle biologicu: Pomacea haustrum Reeve, 1856 (Mollus. ca, Pilidae) sobre planorbíneos, em laboratorio. liev. Saude pübl., S. Paulo, $17: 138-47,1983$.

8. MCANNALY, R.D. \& MOORE, D.V. Predation by the leech Helobdella punctatolineata upon Australorbis glabratus under laboratory conditions. J. Parasit., 52:196-7, 1966.

9. MICHELSON, E.H. Studies on the biological control of schistosome-bearing snails. Predators and parasites of fresh-water molusca: a review of the literature. Parasitology, 47:413-26, 1957. 
GUIMARAES, C.T. et al. Controle biológico: Helobdella triserialis lineata Blanchard, 184: (Hirudinea: Glossiphonidae) sobre Biomphal aria glabrata Say, 1818 (Mollusca: Planorbidae) em laboratório. Rev. Saúde públ., S. Paulo, 17:481-92, 1983.

10. MYIOSFIMA, K. \& JUZEN IGAKKAI, $z$. Lampyrid larva, an anemy of the intermediate host of Schistosoma japonicum. $J$. Perfection Soc., 22:1-42, 1919. In: War. ren, I.S. et al. Schistosomiasis: a bibliography of the world's literature from 1852 to 1962. Ohio, The Press of Western Reserve University, 1967.

11. STORER, T.I. \& USINGER, R.L. General zoology. New York, MCGraw Hill, 1957.
12. YUKI, G. \& KIOTO IGAKKAI, G. Th, intermediate host of Schistosoma japo nicum and the carp. J. Kyoto med. Ass. 16, 1919. In: Warren, K.S. et al. Schisto miasis: a bibliography of the world', literature from 1858 to 1962 . Ohio, Thi Press of Western Reserve University 1967.

Recebido para publicasão em 06/07/1983 Aprovado para publicasão em 01/09/1983 